\title{
Simulated Genital Tract Fluids and Their Applicability in Drug Release/Dissolution Testing of Vaginal Dosage Forms
}

Katharina Tietz and Sandra Klein*

Center of Drug Absorption and Transport (C_DAT), Institute of Biopharmaceutics and Pharmaceutical Technology, Department of Pharmacy, University of Greifswald, Greifswald, Germany

e-mail: Sandra.Klein@uni-greifswald.de

\section{ABSTRACT}

There has been a trend towards the development of novel vaginal dosage forms both for local therapy and systemic absorption. The growing number of vaginal dosage forms, however, presents with an increasing demand for appropriate in vitro test methods for ensuring a safe and reliable in vivo performance of each of the formulations. Application of biorelevant in vitro parameters, i.e., addressing in vivo parameters that significantly affect drug release from a vaginal dosage form in an in vitro test setup, could ensure that the results of the dissolution/drug release test are clinically meaningful and also predictive for detection of changes in the post-approval drug product. Thus, the development of predictive in vitro test methods for vaginal dosage forms is of great interest. A number of simulated vaginal fluids have been introduced in the recent past. In addition, several other genital fluids that could affect the intravaginal fluid composition, such as simulated cervical fluid/mucus, simulated intrauterine fluid, and simulated semen have been described in the literature. More frequent use of these media can be assumed, and future experiments will show if the current media will require more fine-tuning to even better reflect all physiological parameters that can affect drug release/dissolution in the vagina or if there is also a chance to simplify these media without losing their biorelevance.

KEYWORDS: Vaginal fluid, cervicovaginal fluid, uterine fluid, seminal fluid, cervical mucus, dissolution

\section{INTRODUCTION}

Tor or many years, dissolution testing is a routine methodology to provide critical in vitro drug release information for both quality control purposes of solid oral dosage forms and in predicting the in vivo performance of a drug product in formulation development. For the latter purpose, over the past decades, several biorelevant dissolution methodologies addressing physiological parameters relevant to in vivo drug release of orally administered formulation have successfully been developed (1). For many years, the focus of developing biorelevant dissolution test methods was set on obtaining predictive methods for oral dosage forms. However, as a result of a significant technologic advancement in drug delivery that has led to a wider choice of sites for drug administration, the development of predictive in vitro test methods for non-oral drug delivery systems is of great interest. Because it allows for both local and systemic drug delivery, in the recent past, the vagina has witnessed a surge of interest as a site for drug delivery. Dosage forms that can be administered vaginally include classical liquid, semisolid, or solid dosage forms, with creams and gels, suppositories, and tablets being the most commonly used vaginal formulations to date, but also newer types of formulations including, for instance, foams and films and novel delivery systems such as intravaginal rings (IVRs), have been released to the market or can be found in current development pipelines in the pharmaceutical industry.

For many years, vaginal dosage forms (VDFs) were mainly applied for local action in contraception, labor induction, the treatment of vaginal infections, and local menopausal symptoms (2), and the vagina was a rather uncommon route for systemic drug administration. However, it was as early as 1918 when Macht et al. demonstrated that the vaginal epithelium can absorb different substances like alkaloids and inorganic salts (3). These findings were supported by results from other research groups, e.g., in 1947, when Rock et al. could demonstrate that penicillin is easily absorbed from cocoa butter suppositories in the vagina of non-pregnant and post-partum women (4). Nevertheless, even though the large surface area and blood supply of the vaginal epithelium allows for systemic

*Corresponding author 
drug delivery, for many years there were no efforts in developing VDFs for the administration of systemically acting drug compounds.

In the 1960 s several studies focusing on steroid diffusivity through polysiloxane tubes and cylindrical elastomers were performed with regard to obtaining reservoir formulations that could be used as subdermal or intravaginal devices for controlled and long-term drug delivery (e.g. $(5,6)$. In the following years, diffusivity of a large number of steroids, including medroxyprogesterone acetate (MPA), chlormadinone acetate, estradiol, progesterone, levonorgestrel in different elastomers, e.g., polysiloxanes and ethylene vinyl acetate was screened (6-9). In 1968, cylindrical elastomers of polysiloxane impregnated with MPA were fabricated and molded into rings suitable for placement in the vagina (10). It took about 30 years of further development, before in 2001, the first combined (etonogestrel/ethinyl estradiol) contraceptive IVR, the NuvaRing ${ }^{\circledR}$, was approved by the FDA. Since that, the interest in vaginal drug delivery with IVRs has significantly increased. Current trends in IVR development focus on addressing two or more indications from a single product. The overall goal for many of the combined products is to prevent unintended pregnancy and transmission of one or more sexually transmitted infections (STIS) including HIV (11). Because there is currently a common interest in preventing the transmission of STIs, several other platforms are applied for developing VDFs for topical "on demand" prophylaxis for STI transmission or to provide spermicidal action. These include for instance also vaginal films, gels, and foams (12).

The growing number of VDFs presents with an increasing demand for appropriate in vitro test methods for ensuring a safe and reliable in vivo performance of each of the formulations. Application of biorelevant in vitro parameters, i.e., addressing in vivo parameters that significantly affect in vivo drug release from the VDF in an in vitro test setup (fluid volume, $\mathrm{pH}$, buffer capacity, viscosity and surface tension of the physiological fluids and ingredients (such as enzymes) that might affect drug release and stability) could ensure that the results of the dissolution/drug release test are clinically meaningful and also predictive for detection of changes in the postapproval drug product (13). Therefore, establishing biopredictive test methods for VDFs should be of utmost importance for the developer. This review article should give an overview of test media that could be useful for (to a certain extent) simulating the physiological environment in the vagina and that, besides an appropriate instrumental setup, could be applied in the design of biorelevant test methods for VDFs. Moreover, to provide a better understanding of why also other genital fluids might be relevant for designing a biorelevant environment in the dissolution experiment, the anatomy of the female reproductive tract will be briefly discussed.

\section{THE FEMALE REPRODUCTIVE SYSTEM}

The vagina is part of the female reproductive system, which is immature at birth and develops to maturity during puberty. It connects the inner sex organs, i.e., the uterus, the fallopian tubes and the ovaries, with the external sex organs of the vulva (Fig. 1). The vagina itself is a distensible muscular tube which is about $10 \mathrm{~cm}$ long. It serves as entrance to the reproductive tract and as exit from the uterus during menses and childbirth. Because of this particular anatomy, volume and composition of the fluid found in the vaginal cavity do not necessarily reflect the properties and the amount of fluid secreted by the vaginal epithelium but can also contain a certain amount of uterine fluid, cervical mucus and fallopian fluid and, after sexual intercourse, also semen. Therefore, volume and composition of the fluids produced in the female reproductive system are, moreover, impacted by age and stage of the menstrual cycle or pregnancy. Besides that, pathological conditions such as bacterial vaginosis, urinary tract infections, and cancer can impact the vaginal environment. Consequently, when setting up an in vitro test method for a particular VDF, these might also be important aspects to consider.

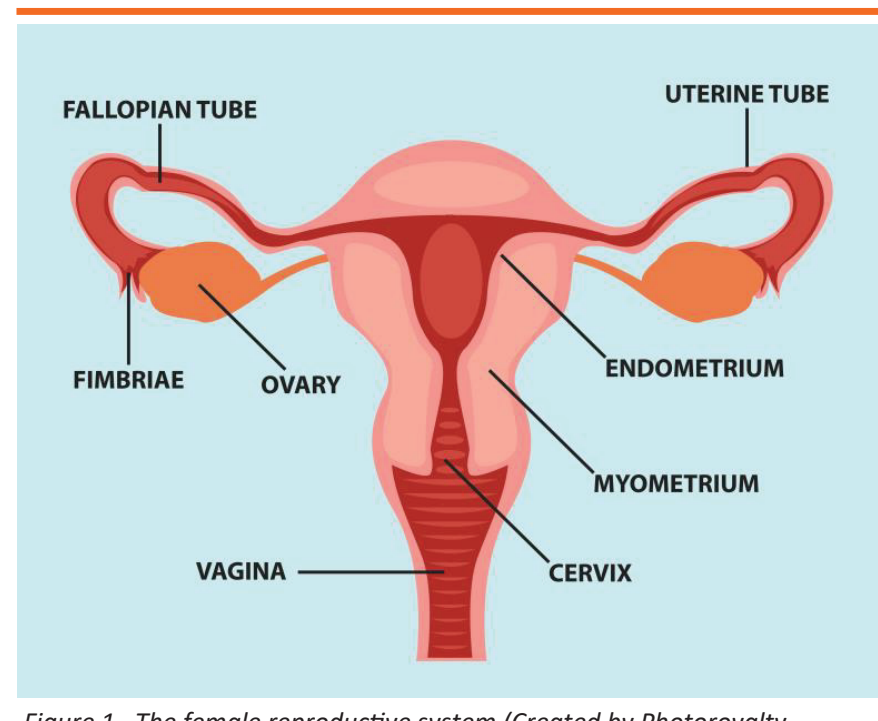

Figure 1. The female reproductive system (Created by Photoroyalty Freepik.com.us).

\section{MEDIA SIMULATING HUMAN VAGINAL FLUID}

The vaginal epithelium does not contain any glands. The fluid entering the vaginal cavity via the vaginal epithelium, i.e., the "real" vaginal fluid, transudates through the 
vaginal epithelium and consists of $90-95 \%$ water. The fluid that can be found in the vagina or is discharged from the vagina, respectively, is a mixture of transudated vaginal fluid with other secretions, such as those of Bartholin's and Skene's glands, which are located close to the vaginal orifice, cervical mucus, endometrial and tubal fluids. In addition, thefluid may also contain someresidual urine and exfoliated epithelium cells. As a consequence, organic and inorganic salts, urea, carbohydrates, mucins, fatty acids, albumin, immunoglobulins, and other macromolecules can be found in vaginal fluid. The main function of the vaginal fluid is to moisten the vaginal epithelium (14). The quantity and composition of human vaginal fluid (HVF) have been studied by various researchers and for different reasons. When screening the literature, it becomes clear that the results obtained in different studies strongly relate to the method of assessing vaginal fluid volumes and properties. Consequently, there is a large variation in the reported fluid volumes that enter the vagina over the duration of $24 \mathrm{~h}$. Owen and Katz reported HVF secretion as $6 \mathrm{~g} /$ day (15), whereas the corresponding secretion rate reported by Paavonen is only 1-3 g/day (14). Wagner and Levin reported a production rate of $0.33 \pm 0.05 \mathrm{~g} / \mathrm{h}$, which would correspond to $7.92 \pm 1.2 \mathrm{~g} /$ day (16). Vaginal resting fluid volumes seem to be less variable and are reported to be in the range of $0.5-0.75 \mathrm{~g}(15)$ or $0.5 \mathrm{~mL}$ (17). As stated before, it should be noted, that both the amount of fluid entering the vagina as well as the vaginal resting fluid volume are subject to inter- and intraindividual variability and are affected by a number of essential parameters like age, phase of the menstrual cycle, and sexual stimulation.

Vaginal fluid $\mathrm{pH}$ in healthy pre-menopausal women is slightly acidic, which is caused by Lactobacilli that represent the most common microorganisms of the healthy vaginal flora. These microbes metabolize glycogen originating from glycogen deposits in vaginal epithelial cells into lactic acid, creating an acidic environment (18). To a lesser extent, lactic acid is, however, also a significant metabolic byproduct of obligate anaerobic glucose metabolism within the vaginal mucosa. Overall, the vaginal $\mathrm{pH}$ is thus determined by the interplay between vaginal physiological processes and microbiology. In reproductive-age women, the average vaginal fluid $\mathrm{pH}$ is 4.5 (19).

With the purpose of closely simulating a typical intravaginal environment for observing the binding and release characteristics of solutions of all-trans-retinoic acid (t-RA), from a collagen sponge diaphragm insert, the first artificial vaginal fluid (AVF, table 1) was developed in 1982 by Dorr et al. (20). The AVF designed for these studies was intended to mimic the electrolyte, nitrogenous, and $\mathrm{pH}$ conditions of a normal HVF in an unstimulated state. Consequently, the media $\mathrm{pH}$ was set to 4.1 , which is in the $\mathrm{pH}$ range of a healthy vaginal environment.

A few years later Geshnizgani and Onderdonk developed a vaginal fluid model (chemically defined medium, CDM) for studying the growth of the vaginal microflora under in vitro test conditions comparable to those in the human vagina (21). The focus of the study was to investigate specific mechanisms and factors that control microbial populations. A key requirement for the CDM was to support the growth of all the bacterial species that make up a healthy human vaginal flora. Consequently, the medium was designed on the basis of published analyses of vaginal secretions. Potassium hydrogen-phosphate and potassium dihydrogen-phosphate were chosen as main buffering agents, cysteine hydrochloride was added as a reducing agent, and Tween 20 was added as a source of long-chain fatty acids and as growth stimulant for Lactobacillus species. Although published reports do not indicate the presence of other growth factors in vaginal fluids, most microorganisms require some additional growth factors in an in vitro environment. Consequently, the CDM was supplemented with a variety of additional growth factors. The preparation of CDM was performed in three separate steps (CDM parts I-III), comprising the preparation and mixing of a variety of stock solutions, before uniting them to the final medium. CDM part I (Tables 1 and 2) was prepared by dissolving the different ingredients in deionized water and adjusting the $\mathrm{pH}$ value to 7.20 with sodium hydroxide. Subsequently, the resulting solution was sterilized in an autoclave $\left(121^{\circ} \mathrm{C}\right.$, $15 \mathrm{~min}$ ). In parallel, the different stock solutions for CDM part II (Table 2) were prepared separately and autoclaved or sterilized by filtration $(0.22 \mu \mathrm{m})$. CDM part III (Table 2) was a commercially available vitamin mixture stock solution. After sterilization and equilibration to room temperature, CDM part I was mixed with appropriate amounts of the stock solutions of CDM part II and the vitamin stock solution of CDM part III to obtain CDM.

In 1999, Owen and Katz developed a vaginal fluid simulant (VFS, Table 1) based on data obtained from an intensive literature review on the composition of HVF (22). After studying of how the physicochemical properties of native vaginal fluid, particularly the $\mathrm{pH}$ and osmolarity, affect the deployment and delivery of contraceptive and prophylactic compounds, VFS was specifically developed for addressing such impacts on in vitro dissolution/drug release testing of VDFs. The medium is intended to model vaginal fluid properties of healthy, non-pregnant and 
Table 1. Simulated Vaginal Fluids

\begin{tabular}{|c|c|c|c|c|c|c|}
\hline Composition & AVF (20) & CDM part I (21) & VFS (22) & MSVF (23) & VFS+G (17) & VFS-G (17) \\
\hline Sodium chloride & $2.279 \mathrm{~g}$ & $3.506 \mathrm{~g}$ & $3.510 \mathrm{~g}$ & $3.500 \mathrm{~g}$ & $4.500 \mathrm{~g}$ & $5.250 \mathrm{~g}$ \\
\hline Potassium hydroxide & - & - & $1.400 \mathrm{~g}$ & - & - & - \\
\hline Calcium hydroxide & - & - & $0.222 \mathrm{~g}$ & - & - & - \\
\hline Albumin/BSA* & $0.009 \mathrm{~g}$ & - & $0.018 \mathrm{~g} *$ & $2.000 \mathrm{~g}$ & - & - \\
\hline Lactic acid & - & - & $2.000 \mathrm{~g}$ & $2.000 \mathrm{~g}$ & $0.610 \mathrm{~g}$ & $0.610 \mathrm{~g}$ \\
\hline Acetic acid & - & - & $1.000 \mathrm{~g}$ & $1.000 \mathrm{~g}$ & $0.79 \mathrm{~mL}$ & $0.79 \mathrm{~mL}$ \\
\hline Glycerol & - & - & $0.160 \mathrm{~g}$ & - & - & - \\
\hline Urea & $0.490 \mathrm{~g}$ & - & $0.400 \mathrm{~g}$ & $0.500 \mathrm{~g}$ & - & - \\
\hline Glucose/Glucose monohydrate** & - & $10.800 \mathrm{~g}$ & $5.000 \mathrm{~g}$ & $10.000 \mathrm{~g}$ & $5.000 \mathrm{~g} * *$ & - \\
\hline Potassium chloride & $1.752 \mathrm{~g}$ & $1.500 \mathrm{~g}$ & - & $1.500 \mathrm{~g}$ & - & - \\
\hline Sodium acetate & $1.805 \mathrm{~g}$ & - & - & - & $0.310 \mathrm{~g}$ & $0.310 \mathrm{~g}$ \\
\hline Amino acids & $0.011 \mathrm{~g}$ & - & - & - & - & - \\
\hline Sodium lactate ( $50 \%$ wt aqueous solution) & - & - & - & - & $4.040 \mathrm{~g}$ & $4.040 \mathrm{~g}$ \\
\hline Potassium hydrogen-phosphate & - & $1.740 \mathrm{~g}$ & - & - & - & - \\
\hline Potassium dihydrogen-phosphate & - & $1.360 \mathrm{~g}$ & - & - & - & - \\
\hline Glycogen & - & - & - & $10.000 \mathrm{~g}$ & - & - \\
\hline Cysteine-HCl & - & $0.500 \mathrm{~g}$ & - & $0.500 \mathrm{~g}$ & - & - \\
\hline Tween 80 & - & - & - & $1.064 \mathrm{~g}$ & - & - \\
\hline Mucin & - & - & - & $0.250 \mathrm{~g}$ & - & - \\
\hline Demineralized water & to $1 \mathrm{~L}$ & to $1 \mathrm{~L}$ & to $1 \mathrm{~L}$ & to $1 \mathrm{~L}$ & to $100 \mathrm{~mL}$ & to $100 \mathrm{~mL}$ \\
\hline pH & 4.10 & 7.20 & 4.20 & 4.25 & 4.20 & 4.20 \\
\hline pH-adjustment with & Acetic acid & $\mathrm{NaOH}$ & $\mathrm{HCl}$ & not specified & Acetic acid & Acetic acid \\
\hline
\end{tabular}

*When indicated, BSA was used instead of albumin; **, when indicated, glucose monohydrate was used instead of glucose; AVF-artificial vaginal fluid; CDM-chemically defined medium; VFS-vaginal fluid simulant; MSVF-medium simulating vaginal fluid; G-glucose; BSA-bovine serum albumin.

premenopausal women. VFS contains lactic and acetic acid to represent the organic acids present in vaginal fluid, and the native lubricating agents of HVF are mimicked by addition of glycerol that is thought to account for the lubricating properties of HVF.

In 2007, Juárez Tomás and Nader-Macías designed a medium simulating vaginal fluid (MSVF, Table 1) to study the impact of vaginal fluid composition and properties on the growth of potentially probiotic Lactobacilli (23). Moreover, the MSVF should help to gain a better understanding of how potentially probiotic microorganisms interact with the native vaginal environment to facilitate the design of vaginal probiotic formulations with improved action. Similar to the CDM proposed by Geshnizgani and Onderdonk, MSVF was prepared stepwise. First, mixture 1 was prepared by dissolving glucose, lactic acid, acetic acid, sodium chloride, potassium chloride, cysteine hydrochloride, and Tween 80 in distilled water. This mixture was autoclaved for 15 minutes at $121{ }^{\circ} \mathrm{C}$ and had a pH of $4.20-4.30$. Mixture 2 was obtained by collating the following aqueous solutions: $50 \mathrm{~mL}$ glycogen $20 \%, 18.8 \mathrm{~mL}$ mucin $1.33 \%, 1.25 \mathrm{~mL}$ urea
$40 \%$, and $40 \mathrm{~mL}$ albumin $5 \%$. Except the albumin solution, all other solutions had been sterilized in an autoclave $\left(121^{\circ} \mathrm{C}, 15 \mathrm{~min}\right.$ ) before mixing. The albumin solution was added after filtration through a $0.22 \mu \mathrm{m}$ filter. In a final step, mixture $1(0.89 \mathrm{~L})$ was added to mixture $2(0.11 \mathrm{~L})$ and the resulting MSVF was stored in the refrigerator until use.

Recently, Rastogi et al. reported the composition of simplified and stable VFS media (sVFS, Table 1) that were designed to mimic the salient physicochemical properties of native vaginal fluids, particularly the buffer capacity and osmolality, in in vitro experiments (17). For designing these media, they had collected HVF and determined its buffer capacity. From the literature they had identified lactate and acetate as the major contributors to buffering capacity and ionic strength. Based on the VFS composition presented by Owen and Katz, they then designed two different sVFS media with fine-tuned ionic strength, osmolality, and buffer capacity. One of the media, VFS+G, contains glucose; the other one, VFS-G, does not. The media were designed as VFS $+G$ and VFS-G $10 x$ stock solutions, which can be easily diluted to the 
Table 2. Complete Composition of CDM (21)

\begin{tabular}{|c|c|c|c|c|}
\hline Composition & Part & $\mathrm{g} / \mathrm{L}$ & $\begin{array}{l}\text { Stock solution } \\
\text { (Volume in } \mathrm{mL} \text { ) }\end{array}$ & Final concentration \\
\hline Sodium chloride & I & 3.50 & - & $0.06 \mathrm{M}$ \\
\hline Potassium chloride & I & 1.50 & - & $0.02 \mathrm{M}$ \\
\hline Potassium hydrogen-phosphate & $\mathrm{I}$ & 1.74 & - & $0.01 \mathrm{M}$ \\
\hline Potassium dihydrogen-phosphate & I & 1.36 & - & $0.01 \mathrm{M}$ \\
\hline Glucose & I & 10.80 & - & $0.06 \mathrm{M}$ \\
\hline Cysteine-HCl & $\mathrm{I}$ & 0.50 & - & $0.003 \mathrm{M}$ \\
\hline Glycogen & II & - & $5.0 \%(20.00)$ & $0.1 \%$ \\
\hline Mucin & II & - & $1.25 \%(20.00)$ & $0.025 \%$ \\
\hline Tween 20 & II & - & $2.0 \%(10.00)$ & $0.02 \%$ \\
\hline Urea & II & - & $40.0 \%(1.25)$ & $0.05 \%$ \\
\hline Vitamin $\mathrm{K}_{1}$ & II & - & $0.5 \%(0.20)$ & $0.01 \%$ \\
\hline Hemin & II & - & $0.5 \%(10.00)$ & $0.05 \%$ \\
\hline Albumin & II & - & $5.0 \%(40.00)$ & $0.2 \%$ \\
\hline Magnesium sulphate & II & - & $6.0 \%(5.00)$ & $0.03 \%$ \\
\hline Sodium hydrogen-carbonate & II & - & $4.0 \%(1.00)$ & $0.004 \%$ \\
\hline Biotin & III & - & - & $0.005 \mathrm{mg} / \mathrm{mL}$ \\
\hline myo-Inositol & III & - & - & $50.0 \mathrm{mg} / \mathrm{mL}$ \\
\hline Niacinamide & III & - & - & $0.5 \mathrm{mg} / \mathrm{mL}$ \\
\hline Pyroxidine $\mathrm{HCl}$ & III & - & - & $0.5 \mathrm{mg} / \mathrm{mL}$ \\
\hline Thiamine $\mathrm{HCl}$ & III & - & - & $0.5 \mathrm{mg} / \mathrm{mL}$ \\
\hline D-Calcium pantothenate & III & - & - & $0.5 \mathrm{mg} / \mathrm{mL}$ \\
\hline Folic acid & III & - & - & $0.5 \mathrm{mg} / \mathrm{mL}$ \\
\hline p-Aminobenzoic acid & III & - & - & $0.01 \mathrm{mg} / \mathrm{mL}$ \\
\hline Choline chloride & III & - & - & $0.5 \mathrm{mg} / \mathrm{mL}$ \\
\hline Riboflavin & III & - & - & $0.1 \mathrm{mg} / \mathrm{mL}$ \\
\hline L-Ascorbic acid & III & - & - & $1.0 \mathrm{mg} / \mathrm{mL}$ \\
\hline Vitamin A (retinol) & III & - & - & $0.005 \mathrm{mg} / \mathrm{mL}$ \\
\hline Vitamin D (Cholecalciferol) & III & - & - & $0.005 \mathrm{mg} / \mathrm{mL}$ \\
\hline Vitamin B12 & III & - & - & $0.1 \mathrm{mg} / \mathrm{mL}$ \\
\hline
\end{tabular}

CDM-chemically defined medium.

final concentration immediately before use. Overall, the media are quite easy to prepare and were found to be stable at $4{ }^{\circ} \mathrm{C}$ for 1 month.

Another medium that is often used to simulate vaginal conditions is acetate buffer, but even though it contains one of the essential organic acids, it does not represent additional components that can be found in HVF. The $\mathrm{pH}$ of acetate buffer can be set in a range of 4.0-4.5. Consequently, the buffer can mimic physiological $\mathrm{pH}$ conditions in the vagina. Moreover, acetate buffer is one of the standard buffers in routine dissolution testing, is easy to prepare, and has quite good storage stability, and therefore, is often the medium of choice in early formulation screening and quality control (QC) of VDFs (24-29).

\section{MEDIA SIMULATING HUMAN}

\section{CERVICOVAGINAL FLUID/MUCUS}

Human cervical mucus serves as a lubricant, is a natural protective barrier, and has a clearance function. The composition of human cervical mucus fluctuates during the menstrual cycle. Main components of human cervicovaginal mucus present at any time in the menstrual cycle are glycoproteins and plasma proteins, other proteins like immunoglobulins, amino acids, cholesterol and other lipids, and inorganic ions. Cervical mucus contains $90-99 \%$ water. For mid-cycle cervical mucus of pre-menopausal women, a $\mathrm{pH}$ of approximately 7.4 was reported $(30,31)$. 
Fresh human cervicovaginal mucus is hard to obtain in large quantities. Therefore, Burruano and colleagues developed a synthetic substitute for human cervicovaginal mucus, i.e. synthetic cervical mucus fluid formulation (SCMF, Table 3) that can be used as a test medium for in vitro experiments in VDF development and screening (31). The medium is intended to simulate both the chemical composition and the physicochemical properties of human cervicovaginal fluid. Essential ingredients of this medium, which are not found in most of the VFS media, are mucin and guar gum to properly adjust media viscosity. Furthermore, the $\mathrm{pH}$ of SCMF is much higher than that of VFS and was set at 7.4 to simulate physiological $\mathrm{pH}$ conditions (31).

Table 3. Simulated Cervicovaginal Fluids

\begin{tabular}{|l|c|c|}
\hline Composition & SCMF (31) & AVF (32) \\
\hline Guar gum & $1.00 \%$ & - \\
\hline Dried porcine gastric mucin (type III) & $0.50 \%$ & $15.0 \mathrm{~g}$ \\
\hline Imidurea & $0.30 \%$ & - \\
\hline Methylparaben & $0.15 \%$ & - \\
\hline Propylparaben & $0.02 \%$ & - \\
\hline Potassium hydrogen-phosphate & $0.26 \%$ & - \\
\hline Potassium dihydrogen-phosphate & $1.57 \%$ & - \\
\hline 0.1 M sodium borate solution & $1 \mathrm{~mL}$ & - \\
\hline Potassium chloride & - & $1.752 \mathrm{~g}$ \\
\hline Sodium chloride & - & $2.279 \mathrm{~g}$ \\
\hline Sodium acetate & - & $1.805 \mathrm{~g}$ \\
\hline Albumin & - & $9.0 \mathrm{mg}$ \\
\hline Amino acids & - & $11.0 \mathrm{mg}$ \\
\hline Glycerol & - & $0.16 \mathrm{~g}$ \\
\hline Urea & - & $0.4 \mathrm{~g}$ \\
\hline Lactic acid & - & $2.0 \mathrm{~g}$ \\
\hline Acetic acid & - & to pH 4.10 \\
\hline Demineralized water & $96.20 \%$ & to $1 \mathrm{~L}$ \\
\hline pH & 7.40 & 4.10 \\
\hline pH-adjustment with & not specified & not specified \\
\hline SCMF-syntetic & & \\
\hline
\end{tabular}

SCMF-synthetic cervical mucus fluid; AVF-artificial vaginal fluid.

Lee et al. used an artificial vaginal fluid (AVF, Table 3) to determine the influence of different mucin concentrations on the viscosity of cervical mucus (32). As expected, the viscosity of AVF increased with increasing mucin concentrations. This observation was also in agreement with the in vivo findings reported by Morales et al., who observed proportional changes of mucin and viscosity levels throughout the menstrual cycle (33).

Overall, there are not many data available on the use of human cervicovaginal fluid/mucus in in vitro dissolution/ drug release testing. Most of the studies reported to date focused on properly mimicking mucus viscosity and how this parameter is affected by the stage of the menstrual cycle and sexual intercourse. With the aim of designing ideal mucus substitutes that are cheap, can be easily prepared, stable, and possess the same sperm penetrability characteristics as human cervical mucus, other groups evaluated hyaluronate solutions, egg white, albumin-containing Tyrode solution, bovine cervical mucus, or other polymer-based systems like polyacrylamide gels as human mucus substitutes. However, as indicated before, even though some of these media can resemble the properties of cervical mucus at certain stages in the menstrual cycle or before and after sexual intercourse, they are unlikely to become frequently used in in vitro dissolution/drug release testing since there are no dosage forms available that are exclusively applied to the cervix or the cervicovaginal region.

\section{MEDIA SIMULATING HUMAN UTERINE FLUID}

Uterine fluid has a complex composition that contains inorganic compounds, carbohydrates, lipid factors, amino acids, peptides, and proteins (34). A simulated uterine fluid (SUF) can be required for in vitro drug release experiments with medicated intrauterine devices (IUDs) or for the investigation of copper corrosion of copperbearing IUDs (Cu-IUDs). The compositions of SUFs described in literature are given in Table 4.

Table 4. Simulated Uterine Fluids

\begin{tabular}{|l|c|c|}
\hline Composition & SUF (35) & SUF (39) \\
\hline Sodium chloride & $4.970 \mathrm{~g}$ & $4.970 \mathrm{~g}$ \\
\hline Potassium chloride & $0.224 \mathrm{~g}$ & $0.224 \mathrm{~g}$ \\
\hline Calcium chloride & $0.167 \mathrm{~g}$ & $0.167 \mathrm{~g}$ \\
\hline Sodium hydrogen-carbonate & $0.250 \mathrm{~g}$ & $0.250 \mathrm{~g}$ \\
\hline Glucose & $0.500 \mathrm{~g}$ & $0.500 \mathrm{~g}$ \\
\hline $\begin{array}{l}\text { Sodium dihydrogen phosphate } \\
\text { dihydrate }\end{array}$ & $0.072 \mathrm{~g}$ & $0.072 \mathrm{~g}$ \\
\hline Urea & - & $0.480 \mathrm{~g}$ \\
\hline Albumin & - & $35.0 \mathrm{~g}$ \\
\hline Demineralized water & to $1 \mathrm{~L}$ & to $1 \mathrm{~L}$ \\
\hline pH & $6.3-8.0$ & $5.0-8.0$ \\
\hline pH-adjustment with & $\mathrm{HCl}$ or $\mathrm{NaOH}$ & $\mathrm{HCl}$ or $\mathrm{NaOH}$ \\
\hline
\end{tabular}

SUF-simulated uterine fluid.

To better estimate the in vivo performance of Cu-IUDs, in 1996 Zhang et al. performed an in vitro study on copper corrosion in SUF (35). Based on reports stating that the chemical and biochemical composition of uterine fluid varies at different phases of the menstrual cycle and from person to person and that uterine fluid shows some 
changes in the presence of an IUD $(36,37)$, Zhang and colleagues wanted to address these variations in their in vitro studies and designed a SUF (Table 4) that qualitatively and quantitatively consisted of the compounds that are commonly available in human uterine fluid (HUF) and that are believed to play an important role in corrosion of Cu-IUD. They varied media $\mathrm{pH}$ (pH 6.3-8.0) and, since there was clinical evidence that oxygen dissolved in the uterine fluid plays an important role in Cu-IUD corrosion, they also varied the oxygen pressure of the media. When evaluating the results of their in vitro studies, they could establish a good correlation between corrosion rate of $\mathrm{Cu}$-IUDS, media $\mathrm{pH}$ and oxygen pressure; however, they unfortunately did not try to establish an in vitro-in vivo correlation.

Zhu et al. also investigated the corrosion behavior of Cu-IUD in a SUF. In contrast to Zhang et al., who did not add any proteins to their SUF, they tried to relate corrosion rate to protein content. For this purpose, they used the SUF designed by Zhang et al., adjusted the $\mathrm{pH}$ to 7.0, added three different proteins (serum albumin, $\gamma$-globulin, and hemoglobin), and varied the total protein concentration of the medium in a range of $0-8 \mathrm{~g} / \mathrm{L}$ in their experiments. They could show that the proteins studied enhanced the corrosion of copper in SUF. Results of the study fit some general trends observed in vivo, but the authors clearly stated that the reported in vitro corrosion rates might not be the same as those obtained from in vivo experiments (38).

With the aim of a detailed investigation of the mechanism of copper corrosion in uterine fluid, Mora et al. studied corrosion of $\mathrm{Cu}$-IUD in simulated uterine fluids of different $\mathrm{pH}(\mathrm{pH} \mathrm{5.0-8.0)}$ and oxygen pressures (0.0-0.20 atmospheres) and varied the test duration within a range of 1-30 days (39). Moreover, they investigated if the presence of urea and/or proteins in SUF will change the corrosion mechanism. Results of their study confirmed the observations made by Zhang et al., since also in the Mora study copper corrosion depended $\mathrm{pH}$ and oxygen pressure of SUF. Moreover, both urea and albumin had a $\mathrm{pH}$-related impact on corrosion rate.

A number of additional authors reported the use of SUFs in studying copper, zinc, or indometacin release from IUDs (40-47). However, in all of these studies, the media designed by Zhang et al. (35) or the slightly modified version reported by Mora et al. (39) were applied. In vivo uterine fluid enters the vagina via the cervix and mixes with the fluid secreted from the vaginal epithelium; therefore, to date, SUF has never been applied for studying drug release of VDFs as a stand-alone medium. However, its composition might become important when the intention is to compose a vaginal medium that addresses the composition of a physiologically relevant mixture of all fluids that enter the vagina and consequently may affect drug release of VDFs.

\section{MEDIA SIMULATING HUMAN SEMINAL FLUID}

During sexual intercourse, HVF mixes with seminal fluid. Human semen is a mixture of components produced by several different glands. During ejaculation, the different components are incompletely mixed. Since the different portions of the ejaculate have different origins, and the secretions of the organs contributing to the ejaculate differ in composition, the ejaculate is not an entirely homogeneous mixture. Overall, the fluid contains acid phosphatase, citric acid, inositol, calcium, zinc, magnesium, fructose, ascorbic acid, prostaglandins, L-carnitine, and neutral alpha-glucosidase, and several additional components (48). The average human ejaculate volume was reported as $3.4 \mathrm{~mL}$ (48). Human seminal fluid (HSF) is slightly alkaline ( $\mathrm{pH}$ 7.7) (48) and has a very high buffering capacity (25 slyke) (48). Its (average) osmolality was reported as $354 \mathrm{mOsmol} / \mathrm{kg}$ (48) or in the range of $360-380 \mathrm{mOsmol} / \mathrm{kg}$, respectively (17). It is obvious that these properties of seminal fluid have an impact on the vaginal environment. Consequently $\mathrm{pH}$, but also additional physicochemical properties of the mixture of vaginal and seminal fluid, should be considered when the aim is to develop vaginally applied formulations with a drug release/dissolution mechanism that can be affected by $\mathrm{pH}$ and other properties of the vaginal environment and that will be present in the vagina during sexual intercourse (e.g. IVRs, gels containing microbicides, etc.). Table 5 gives an overview of simulating seminal fluids described in literature.

Based upon an extensive literature review on the constituents of human semen, in 2005 Owen and Katz proposed a semen fluid simulant (SFS, Table 5) intended to be used for in vitro drug release/dissolution testing of VDFs (48). In choosing the ingredients for this medium, a particular focus was set on achieving physiological properties that might have an impact on drug release/ dissolution of VDFs. Among these properties, $\mathrm{pH}$ and buffering capacity, osmolarity, ionic strength, and rheological properties usually play dominant roles in the physicochemical processes that govern drug release kinetics and delivery vehicle distribution. Even though it was not possible to match the concentration of every 
Table 5. Simulated Seminal Fluids

\begin{tabular}{|c|c|c|c|c|}
\hline Composition & SFS (48) & sSFS+F (17) & sSFS-F (17) & ASF (49) \\
\hline Sodium dihydrogen-phosphate monohydrate & $0.093 \mathrm{~g}$ & $0.97 \mathrm{~g}$ & $0.97 \mathrm{~g}$ & - \\
\hline Sodium hydrogen phosphate & $0.858 \mathrm{~g}$ & $9.46 \mathrm{~g}$ & $9.46 \mathrm{~g}$ & - \\
\hline Sodium citrate dehydrate & $0.813 \mathrm{~g}$ & - & - & $8.13 \mathrm{~g}$ \\
\hline Potassium chloride & $0.091 \mathrm{~g}$ & - & - & $0.432 \mathrm{~g}$ \\
\hline Potassium hydroxide & $0.088 \mathrm{~g}$ & - & - & - \\
\hline D(-)-Fructose & $0.272 \mathrm{~g}$ & $3.64 \mathrm{~g}$ & - & $2.72 \mathrm{~g}$ \\
\hline D-Glucose anhydrous/monohydrate* & $0.102 \mathrm{~g}$ & - & - & $1.12 \mathrm{~g} *$ \\
\hline L(+)-Lactic acid & $0.062 \mathrm{~g}$ & $0.62 \mathrm{~g}$ & $0.62 \mathrm{~g}$ & - \\
\hline Urea & $0.045 \mathrm{~g}$ & - & - & $0.72 \mathrm{~g}$ \\
\hline BSA (fraction V)/HSA* & $5.04 \mathrm{~g}$ & $50.4 \mathrm{~g}$ & - & $11.0 \mathrm{~g} *$ \\
\hline Calcium chloride dihydrate & $0.101 \mathrm{~g}$ & - & - & $0.73 \mathrm{~g}$ \\
\hline Magnesium chloride hexahydrate & $0.092 \mathrm{~g}$ & - & - & - \\
\hline Zinc chloride & $0.034 \mathrm{~g}$ & - & - & - \\
\hline Sodium chloride & - & - & $0.46 \mathrm{~g}$ & $2.69 \mathrm{~g}$ \\
\hline Sodium pyruvate & - & - & - & $0.374 \mathrm{~g}$ \\
\hline Sodium lactate & - & - & - & $0.779 \mathrm{~g}$ \\
\hline Sodium hydrogen carbonate & - & - & - & $2.1 \mathrm{~g}$ \\
\hline Urate & - & - & - & $0.07 \mathrm{~g}$ \\
\hline Ascorbic acid & - & - & - & $0.1 \mathrm{~g}$ \\
\hline Magnesium sulphate & - & - & - & $0.54 \mathrm{~g}$ \\
\hline Zinc sulphate heptahydrate & - & - & - & $0.5 \mathrm{~g}$ \\
\hline Gentamycin & & & & $40 \mathrm{mg}$ \\
\hline Methyl cellulose & - & - & - & $0.8 \%$ \\
\hline Demineralized water & to $100 \mathrm{~mL}$ & to $100 \mathrm{~mL}$ & to $100 \mathrm{~mL}$ & to $1 \mathrm{~L}$ \\
\hline $\mathrm{pH}$ & 7.70 & 7.70 & 7.70 & Not specified \\
\hline Osmolality [mOsmol/kg] & 354 & $364 \pm 1.7$ & $364 \pm 1.7$ & $320 \pm 10$ \\
\hline pH-adjustment with & $\mathrm{NaOH}$ & & & \\
\hline
\end{tabular}

*When indicated, D-Glucose monohydrate was used instead of D-Glucose anhydrous or HSA was used instead of BSA (fraction V);

SFS,-simulated semen fluid; sSFS-simplified simulated semen fluid; $F$, fructose.

component and every physical parameter to the literature values, Owen and Katz tried to mimic the physiological properties as closely as possible.

In 2016, Rastogi et al. redesigned the SFS developed by Owen and Katz (48) and introduced two simplified SFS media (sSFS, Table 5). Analogous to the procedure reported for the sVFS, the SSFS should mimic the salient physicochemical properties of native seminal fluid, particularly the buffer capacity and osmolality, and were designed to be applicable for a variety of in vitro experiments (17). As a first step, Rastogi et al. collected HSF and determined the buffer capacity. Then, they identified the major buffering species from published compositions of reproductive tract fluids. In the next step, ionic strength, buffering capacity, $\mathrm{pH}$, and osmolalities of HSF were calculated or experimentally determined. Based on the SFS composition presented by Owen and Katz, they then designed two different sSFS media with finetuned ionic strength, osmolality, and buffer capacity. One medium, i.e. $S F S+F$, contains fructose and proteins; the other, SFS-F, does not. The media were designed as SFS+F and SFS-F 10x stock solution, which, as described for SVFS, can be easily diluted to their original concentration before use. Similar to sVFS, the media are easy to prepare and stable at $4{ }^{\circ} \mathrm{C}$ for 1 month.

Finally, with the aim of evaluating an artificial culture medium similar to seminal plasma with regard to its effect on the fine structure of human spermatozoa postvitrification, Agha-Rahimi et al. developed an artificial seminal fluid (ASF) based on the recipe of Owen and Katz (49). For their objective, they slightly modified the Owen and Katz SFS medium by adding antioxidants, changing 
the main buffering species, and adjusting the osmolality. Bicarbonate was chosen as the main buffering species and the osmolality was adjusted to $320 \pm 10 \mathrm{Osmol} / \mathrm{kg}$ by adding an appropriate amount of sodium chloride. Further, ascorbic acid, urate, and albumin were added to ASF as antioxidants. Finally, amongst other compounds, the medium contains glucose, fructose, lactate, and pyruvate, which are also naturally present in HSF as sources of energy.

\section{DISCUSSION}

The fluid available for drug release/dissolution in the human vagina is a mixture of fluids from different sources, such as glands located close to the vaginal orifice, cervix, endometrium, and fallopian tubes. Consequently, it contains numerous ingredients, and the composition and volume of HVF available in the human vagina at a given time point are subject to a high intra- and interindividual variability. Thus, the development of predictive in vitro test methods for VDFs is a challenge.

As discussed, several simulated vaginal fluids, also known as vaginal fluid simulants, have been introduced in the recent past. In addition, several other genital fluids that could affect the intravaginal fluid composition, such as simulated cervical fluid/mucus, simulated intrauterine fluid, and simulated semen have been described in the literature. It should be noted that many of these media were not (exclusively) developed for drug release/ dissolution testing and thus may not address all attributes of HVF that are relevant for drug release from vaginal drug formulations. Thus, the selection of an appropriate test medium should be made on a case-by-case basis. However, for such a decision, a detailed understanding of the physiological conditions in the target patient group and the purpose of the experiment are essential. Whereas for many dosage forms, a detailed simulation of various factors such as fluid volume, secretion rate, and discharge, composition, and physicochemical properties of HVF are important to consider when designing biopredictive test methods. The development of appropriate QC methods presents with different challenges such as for instance, how to maintain sink conditions.

Since the resting fluid volume in the human vagina is rather small, particularly for formulations containing poorly soluble compounds, the use of physiological fluid volumes and physiologically based media is usually not feasible in QC of VDFs. When screening drug release from IVRs, i.e. devices that release the active pharmaceutical ingredient (API) over a duration of several weeks or even months, microbial stability of the media is another important issue that is usually not considered when designing physiologically relevant test media. The composition and physicochemical properties of many of the simulated genital fluids discussed in the present manuscript provide excellent conditions for the growth of undesired bacteria and molds when the test duration markedly exceeds $\sim 24 \mathrm{hrs}$. When screening drug release from IVRs, the test medium must be completely replaced in short intervals or a preservative must be added to the medium. In the latter case, the preservative needs to be compatible with all other media ingredients and the formulation to be screened, i.e., it must not affect media properties and drug release or dissolution.

Most simulated genital fluids have a complex composition. Several ingredients, for instance, albumin, a protein that can also be found in human plasma and is well known to bind to numerous drug molecules (plasma protein binding) including several steroid hormones that are also applied in VDFs for contraceptive use, can significantly affect the concentration of free (dissolved) API in the test medium that is accessible by standard analytical techniques, such as UV spectrophotometry. Another problem that presents with media containing albumin or other proteins is protein precipitation in a non-aqueous or less-hydrophilic environment. This can be an issue, when samples need to be filtered before analysis. This situation can occur when preparing samples for liquid chromatography (LC) analysis, which often includes a step where aqueous samples from the release experiment are diluted with an organic solvent before injection. Precipitation can be an issue when samples need to be filtered before analysis (filter clogging), but also during sample analysis when the media composition is altered during analysis, e.g., by applying a solvent gradient in an LC run (column clogging). For these reasons, analytical methods applied in in vitro experiments with complex simulated genital fluids need to be properly validated to also capture API that might be bound to proteins or other ingredients of the test media after release/dissolution and to prevent technical problems during analysis. Due to the complex composition of simulated genital fluids, the development of appropriate analytical methods can be challenging, but these media can allow for simulating a variety of vaginal conditions in healthy and diseased pre- to post-menopausal women including the simulation of the vaginal milieu after sexual intercourse. The latter aspect is important for the evaluation of VDFs that stay in the vagina for several weeks (e.g. IVRs) or are inserted into the vagina immediately before sexual intercourse (e.g. vaginal gels). For simulating such conditions, an 
appropriate in vitro test setup will need to implement the mixture of VFS and SFS after simulated intercourse. Such procedure is of particular importance when designing in vitro test methods for VDFs with contraceptive, microbicide, or spermicide action, which should not only exhibit a robust drug release in VFS, but also when physiological volumes of (simulated) vaginal fluid and (simulated) semen are mixed in vivo, which is usually accompanied by a temporary $\mathrm{pH}$ shift and an increase in buffer capacity of the vaginal contents. This shift can for instance alter the dissolution of drugs with $\mathrm{pH}$-dependent solubility and/or the texture of vaginal gel formulations with $\mathrm{pH}$-dependent viscosity (e.g. polyacrylate gels).

The design of both biopredictive test methods and QC tests for VDFs is not an easy task. The set of simulated genital fluids available to date is a reasonable platform for developing test methods for different VDFs, but it is also clear that there are still significant knowledge gaps regarding which physiological factors determine systemic and local bioavailability of APIs after application of different types of VDFs. Thus, it is more than likely that the overall design of in vitro test methods for VDFs, and particularly the composition and properties of the simulated genital fluids, might undergo sensible finetuning in the design of both QC and biopredictive studies.

\section{CONCLUSION}

A variety of simulated genital fluids are available for a range of different in vitro experiments. Some of these media have already been applied in in vitro release/ dissolution experiments, and others have not because researchers may not be aware of their existence or the media composition did not fit with the design or clinical significance of the experiment. However, with the increasing number of VDFs in current development pipelines, the simulated genital fluids available represent a promising platform for designing biopredictive test methods for VDFs. A more frequent use of these media can be assumed, and future experiments will show if the current media will require more fine-tuning to even better reflect all physiological parameters that can affect drug release/dissolution in the vagina or if there is also a chance to simplify these media without losing their biorelevance.

\section{ACKNOWLEDGEMENTS}

The authors disclosed no funding related to this article.

\section{CONFLICT OF INTEREST}

The authors disclosed no conflicts of interest related to this article.

\section{REFERENCES}

1. Kostewicz, E. S.; Abrahamsson, B.; Brewster, M.; Brouwers, J.; Butler, J.; Carlert, S.; Dickinson, P. A.; Dressman, J.; Holm, R.; Klein, $\mathrm{S}$. In vitro models for the prediction of in vivo performance of oral dosage forms. Eur. J. Pharm. Sci. 2014, 57, 342-366. DOI: 10.1016/j.ejps.2013.08.024.

2. Dodou, K. Exploring the unconventional routes - rectal and vaginal dosage formulations. Pharm. J. 2012, 289, 238.

3. Macht, D. I. On the absorption of drugs and poisons through the vagina. J. Pharmacol. Exp. Ther. 1918, 10, 509-522.

4. Rock, J.; Barker, R. H.; Bacon, W. B. Vaginal absorption of penicillin. Science 1947, 105, 13. DOI: 10.1126/science.105.2714.13.

5. Folkmann, J.; Long, D. M. The use of silicone rubber as a carrier for prolonged drug therapy. J. Surg. Res. 1964, 4, 139-142. DOI: 10.1016/S0022-4804(64)80040-8.

6. Dziuk, P. J.; Cook, B. Passage of steroids through silicone rubber. Endocrinology 1966, 78, 208-211. DOI: 10.1210/endo-78-1-208.

7. Mishell, D. R. Intravaginal rings for contraceptive use. Contraception 1975, 12, 249-251. DOI: 10.1016/00107824(75)90085-2.

8. Malcolm, K.; Woolfson, D.; Russell, J.; Tallon, P.; McAuley, L.; Craig, D. Influence of silicone elastomer solubility and diffusivity on the in vitro release of drugs from intravaginal rings. J. Control. Release 2003, 90, 217-225. DOI: 10.1016/S01683659(03)00178-0.

9. van Laarhoven, J. A.; Kruft, M. A.; Vromans, H. In vitro release properties of etonogestrel and ethinyl estradiol from a contraceptive vaginal ring. Int. J. Pharm. 2002, 232, 163-173. DOI: 10.1016/S0378-5173(01)00900-0.

10. Mishell, D. R.; Talas, M.; Parlow, A. F.; Moyer, D. L. Contraception by means of a Silastic vaginal ring impregnated with medroxyprogesterone acetate. Am. J. Obstet. Gynecol. 1970, 107, 100-107. DOI: 10.1016/S0002-9378(16)33897-2.

11. Friend, D. R. An update on multipurpose prevention technologies for the prevention of HIV transmission and pregnancy. Exp. Opin. Drug Deliv. 2016, 13, 533-545. DOI: 10.1517/17425247.2016.1134485.

12. Patel, S. K.; Rohan, L. C. On-demand microbicide products: design matters. Drug Deliv. Transl. Res. 2017, 7, 775-795. DOI: 10.1007/s13346-017-0385-4.

13. Biswas, D.; Halquist, M. Using biorelevant in vitro models testing to characterize release of non oral dosage forms as another tool for safety. J. Pharmacovigilance 2016, 4, e153. DOI: 10.4172/2329-6887.1000e153.

14. Paavonen, J. Physiology and ecology of the vagina. Scand. J. Infect. Dis. 1983, 40, 31-35.

15. Owen, D. H.; Katz, D. F. A vaginal fluid simulant. Contraception 1999, 59, 91-95. DOI: 10.1016/S0010-7824(99)00010-4.

16. Wagner, G.; Levin, R. J. Electrolytes in vaginal fluid during the menstrual-cycle of coitally active and inactive women. J. Reprod. Fertil. 1980, 60, 17-27. DOI: 10.1530/jrf.0.0600017. 
17. Rastogi, R.; Su, J.; Mahalingam, A.; Clark, J.; Sung, S.; Hope, T.; Kiser, P. F. Engineering and characterization of simplified vaginal and seminal fluid simulants. Contraception 2016, 93, 337-346. DOI: 10.1016/j.contraception.2015.11.008.

18. Mirmonsef, P.; Hotton, A. L.; Gilbert, D.; Burgad, D.; Landay, A,; Weber, K. M.; Cohen, M.; Ravel, J.; Spear, G. T. Free glycogen in vaginal fluids is associated with lactobacillus colonization and low vaginal pH. PLoS One 2014, 9, e102467. DOI: 10.1371/ journal.pone.0102467.

19. Linhares, I. M.; Summers, P. R.; Larsen, B.; Giraldo, P. C.; Witkin, S. S. Contemporary perspectives on vaginal $\mathrm{pH}$ and lactobacilli. Am. J. Obstet. Gynecol. 2011, 204, 120.e1-5. DOI: 10.1016/j. ajog.2010.07.010.

20. Dorr, R. T.; Surwit, E. A.; Droegemueller, W.; Alberts, D. S.; Meyskens, F. L.; Chvapil, M. In vitro retinoid binding and release from a collagen sponge material in a simulated intravaginal environment. J. Biomed. Mater. Res. 1982, 16, 839-850. DOI: 10.1002/jbm.820160609.

21. Geshnizgani, A. M.; Onderdonk, A. B. Defined medium simulating genital-tract secretions for growth of vaginal microflora. J. Clin. Microbiol. 1992, 30, 1323-1326.

22. Owen, D. H.; Katz, D. F. A vaginal fluid simulant. Contraception 1999, 59, 91-95. DOI: 10.1016/S0010-7824(99)00010-4.

23. Juárez Tomás, M. S.; Nader-Macías, M. E. Effect of a medium simulating vaginal fluid on the growth and expression of beneficial characteristics of potentially probiotic lactobacilli. In Communicating Current Research and Educational Topics and Trends in Applied Microbiology; Méndez-Vilas, A., Ed.; Formatex, 2007, pp 732-739.

24. Externbrink, A.; Clark, M. R.; Friend, D. R.; Klein, S. Investigating the feasibility of temperature-controlled accelerated drug release testing for an intravaginal ring. Eur. J. Pharm. Biopharm. 2013, 85, 966-973. DOI: 10.1016/j.ejpb.2013.06.004.

25. Externbrink, A.; Eggenreich, K.; Eder, S.; Mohr, S.; Nickisch, K.; Klein, S. Development and evaluation of accelerated drug release testing methods for a matrix-type intravaginal ring. Eur. J. Pharm. Biopharm. 2017, 110, 1-12. DOI: 10.1016/j.ejpb.2016.10.012.

26. Teller, R. S.; Malaspina, D. C.; Rastogi, R.; Clark, J. T.; Szleifer, I.; Kiser, P. F. Controlling the hydration rate of a hydrophilic matrix in the core of an intravaginal ring determines antiretroviral release. J. Control. Release 2016, 224, 176-183. DOI: 10.1016/j. jconrel.2015.12.035.

27. Traore, Y. L.; Chen, Y.; Bernier, A. M.; Ho, E. A. Impact of hydroxychloroquine-loaded polyurethane intravaginal rings on lactobacilli. Antimicrob. Agents Chemother. 2015, 59, 76807686. DOI: 10.1128/AAC.01819-15.

28. Clark, J. T.; Johnson, T. J.; Clark, M. R.; Nebeker, J. S.; Fabian, J.; Tuitupou, A. L.; Ponnapalli, S.; Smith, E. M.; Friend, D. R.; Kiser, P. F. Quantitative evaluation of a hydrophilic matrix intravaginal ring for the sustained delivery of tenofovir. J. Control. Release 2012, 163, 240-248. DOI: 10.1016/j.jconrel.2012.08.033.
29. Johnson, T. J.; Clark, M. R.; Albright, T. H.; Nebeker, J. S.; Tuitupou, A. L.; Clark, J. T.; Fabian, J.; McCabe, R. T.; Chandra, N.; Doncel, G. F. A 90-day tenofovir reservoir intravaginal ring for mucosal HIV prophylaxis. Antimicrob. Agents Chemother. 2012, 56, 62726283. DOI: 10.1128/AAC.01431-12.

30. Tang, S.; Garrett, C.; Baker, H. W. Comparison of human cervical mucus and artificial sperm penetration media. Hum. Reprod. 1999, 14, 2812-2817. DOI: 10.1093/humrep/14.11.2812.

31. Burruano, B. T.; Schnaare, R. L.; Malamud, D. Synthetic cervical mucus formulation. Contraception 2002, 66, 137-140. DOI: 10.1016/S0010-7824(02)00336-0.

32. Lee, C. H.; Wang, Y.; Shin, S. C.; Chien, Y. W. Effects of chelating agents on the rheological property of cervical mucus. Contraception 2002, 65, 435-440. DOI: 10.1016/S00107824(02)00300-1.

33. Morales, P.; Roco, M.; Vigil, P. Human cervical mucus: relationship between biochemical characteristics and ability to allow migration of spermatozoa. Hum. Reprod. 1993, 8, 78-83. DOI: 10.1093/oxfordjournals.humrep.a137879.

34. Hannan, N. J.; Nie, G.; Rainzcuk, A.; Rombauts, L. J.; Salamonsen, L. A. Uterine lavage or aspirate: which view of the intrauterine environment? Reprod. Sci. 2012, 19, 1125-1132. DOI: 10.1177/1933719112443879.

35. Zhang, C.; Xu, N.; Yang, B. The corrosion behaviour of copper in simulated uterine fluid. Corros. Sci. 1996, 38, 635-641. DOI: 10.1016/0010-938X(95)00154-C.

36. Kar A. B.; Engineer, A. D.; Goel, R.; Kamboj, V. P.; Dasgupta, P. R.; Chowdhury, S. R. Effect of an intrauterine contraceptive device on biochemical composition of uterine fluid. Am. J. Obstet. Gynecol. 1968, 101, 966-970. DOI: 10.1016/0002-9378(68)90283-4.

37. Sedlis, A.; Kandemir, E.; Stone, M. L. Intrauterine $\mathrm{pH}$ of women using stainless steel contraceptive device. Obstet. Gynecol. 1967, 30, 114-117.

38. Zhu, J.; Xu, N.; Zhang, C. Characteristics of copper corrosion in simulated uterine fluid in the presence of protein. Adv. Contracept. 1999, 15, 179-190. DOI: 10.1023/A:1006793231274.

39. Mora, N.; Cano, E.; Mora, E. M.; Bastidas, J. M. Influence of $\mathrm{pH}$ and oxygen on copper corrosion in simulated uterine fluid. Biomaterials 2002, 23, 667-671. DOI: 10.1016/S01429612(01)00154-5.

40. Bastidas, J. M.; Mora, N.; Cano, E.; Polo, J. L. Characterization of copper corrosion products originated in simulated uterine fluids and on packaged intrauterine devices. J. Mater. Sci. Mater. Med. 2001, 12, 391-397. DOI: 10.1023/A:1011288701499.

41. Bastidas, D. M.; Cano, E.; Mora, E. M. Influence of oxygen, albumin and $\mathrm{pH}$ on copper dissolution in a simulated uterine fluid. Eur. J. Contracep. Reprod. Health Care 2005, 10, 123-130. DOI: 10.1080/13625180500131642.

42. Arancibia, V.; Pena, C.; Allen, H. E.; Lagos, G. Characterization of copper in uterine fluids of patients who use the copper T-380A intrauterine device. Clin. Chim. Acta. 2003, 332, 69-78. DOI: 10.1016/S0009-8981(03)00124-4. 
43. Jinying, L.; Ying, L.; Xuan, G.; Yanli, G.; Jianping, L. Investigation of the release behavior of cupric ion for three types of $\mathrm{Cu}$ IUDs and indomethacin for medicated Cu-IUD in simulated uterine fluid. Contraception 2008, 77, 299-302. DOI: 10.1016/j. contraception.2007.12.005.

44. Zhou, X.; Li, Y.; Jiang, X.; Qiu, L.; Liu, J. Release of copper and indomethacin from intrauterine devices immersed in simulated uterine fluid. Eur. J. Contracep. Reprod. Health Care 2010, 15, 205-212. DOI: 10.3109/13625181003782860.

45. Yang, Z. H.; Xie, C.; Xia, X.; Cai, S. Zn(2+) release behavior and surface characteristics of $\mathrm{Zn} / \mathrm{LDPE}$ nanocomposites and $\mathrm{ZnO} /$ LDPE nanocomposites in simulated uterine solution. J. Mater. Sci. Mater. Med. 2008, 19, 3319-3326. DOI: 10.1007/s10856008-3467-5.

46. Zhang, S.; Li, Y.; Yu, P.; Chen, T.; Zhou, W. S.; Zhang, W.; Liu, J. In vitro release of cupric ion from intrauterine devices: influence of frame, shape, copper surface area and indomethacin. Biomed. Microdevices 2015, 17, 19. DOI: 10.1007/s10544-014-9924-7.

47. Alonso, C.; Casero, E.; Roman, E.; Campos, S. F-P.; de Mele, M. F. L. Effective inhibition of the early copper ion burst release by purine adsorption in simulated uterine fluids. Electrochim. Acta. 2016, 189, 54-63. DOI: 10.1016/j.electacta.2015.12.093.

48. Owen, D. H.; Katz, D. F. A review of the physical and chemical properties of human semen and the formulationo a semen simulant. J. Androl. 2005, 26, 459-469. DOI: 10.2164/ jandrol.04104.

49. Agha-Rahimi, A.; Khalili, M. A.; Nottola, S. A.; Miglietta, S.; Moradi, A. Cryoprotectant-free vitrification of human spermatozoa in new artificial seminal fluid. Andrology 2016, 4, 1037-1044. DOI: 10.1111/andr.12212. 